\title{
Asymmetric Carrier Penetration into Hexagonal Boron Nitride in Graphene
}

\section{Field-Effect Transistors}

Susumu Okada, ${ }^{1, a)}$ Mina Maruyama, ${ }^{1}$ and Yanlin Gao ${ }^{1}$

Department of Physics, Graduate School of Pure and Applied Sciences,

University of Tsukuba, 1-1-1 Tennodai, Tsukuba, Ibaraki 305-8571,

Japan

The electronic structure of graphene field-effect transistors with a hexagonal boron nitride (h-BN) gate dielectric was studied in terms of the gate electric field and dielectric thickness using the density functional theory combined with the effective screening medium method. The calculation results showed that the carrier penetration into monolayer h-BN depended on the gate voltage that injected electrons or holes into graphene. The critical voltage for hole penetration was lower than that for electron penetration. At a fixed carrier concentration, carrier penetration soon occurred with increasing h-BN thickness for hole doping.

a)E-mail address: sokada@comas.frsc.tsukuba.ac.jp 
Its hexagonal covalent network composed of $\mathrm{sp}^{2}$ carbon atoms endows graphene with unusual physical properties that are applicable in a wide range of modern technologies ${ }^{1,2}$. Because of a conical dispersion band at the Fermi level ${ }^{3-5}$, the ultimate two-dimensional electron system of graphene leads to an unusual quantum Hall effect and results in a remarkably high carrier mobility of a few hundred thousand centimeters squared per volt seconds ${ }^{6-8}$. These features make graphene a unique and attractive material for various state-of-the-art devices, such as semiconducting devices, sensors, and transparent electrodes. However, it is difficult to fabricate graphene-based devices because the electronic structure of graphene is fragile against the formation of hybrid structures with other materials. Vacancies ${ }^{9-12}$, topological defects ${ }^{13-15}$, edges ${ }^{16-18}$, and atomic/molecular adsorbates ${ }^{19,20}$ intrinsically modulate the characteristic conical bands of isolated freestanding graphene. Most foreign materials open the band gap of graphene; the extent to which they do this strongly depends on their morphology and concentration. In addition to foreign materials, other layers of graphene also affect its conical band and lead to variation of the band structure depending on the interlayer stacking arrangement ${ }^{21-24}$.

In semiconducting devices containing graphene, hybrid structures composed of graphene and dielectric substrates $\left(\mathrm{SiO}_{2}\right.$ and $\left.\mathrm{Al}_{2} \mathrm{O}_{3}\right)$ are essential. In such hybrid structures, the surface roughness of dielectrics and charged impurities on their surfaces cause serious deterioration of graphene mobility ${ }^{25-29}$, because the structural deformation of graphene changes the band structure and impurities act as scattering centers for the conducting carriers on graphene. Furthermore, spatial undulation of the electrostatic potential of graphene by dielectric surfaces leads to a finite energy gap between bonding and antibonding $\pi$ states of graphene, even when the dielectrics possess perfectly flat and clean surfaces ${ }^{30,31}$. Hexagonal boron nitride (h-BN) is a possible dielectric material that can overcome these difficulties because of its atomically flat two-dimensional ridged network with insulating electronic structure. Indeed, remarkable carrier mobility has been observed for graphene adsorbed on h-BN ${ }^{32}$. However, the ultimate thickness of h-BN causes another problem when fabricating graphene-based devices. The layered structure of h-BN might be a shortcoming in terms of its lower gate dielectric constant than those of the other conventional gate dielectrics, even though it is an insulator with a wide band gap of over $5 \mathrm{eV}^{33,34}$.

Although h-BN can work as an insulating layer for graphene devices, theoretical insight into the dielectric strength of such devices in terms of thickness and carrier concentration 
is still lacking. In particular, it is important to investigate the possibility of the charge accumulation in dielectric layers under the electric field, because such charge accumulation occasionally causes the soft dielectric breakdown. Thus, in this work, we aim to investigate the dielectric properties of h-BN in graphene field-effect transistors (FETs) using a combination of the density functional theory (DFT) and effective screening medium (ESM) method. The calculation results demonstrate the asymmetric carrier penetration into h-BN thin films in terms of the gate voltage. The carrier penetration upon a positive gate voltage (electron doping) is easier than that upon a negative gate voltage (hole doping), which is ascribed to the band alignment between graphene and h-BN.

All calculations were conducted using the DFT ${ }^{35,36}$ implemented in the STATE package ${ }^{37}$. We used the local density approximation (LDA) with the Perdew-Zunger functional ${ }^{38,39}$ to describe the exchange-correlation potential energy between interacting electrons, because the LDA is known to reproduce the interlayer stacking of graphene- and h-BN-related materials ${ }^{40,41}$. Ultrasoft pseudopotentials generated by the Vanderbilt scheme were used to describe the electrons-ion interactions ${ }^{42}$. The valence wave functions and deficit charge density were expanded using the plane-wave basis set with cutoff energies of 25 and 225 Ry, respectively. We adopted the ESM method to solve the generalized Poisson equation along normal to graphene and h-BN layers, to consider an external electric field and excess carriers $^{43,44}$. The Brillouin-zone integration was carried out with $20 \times 20 \times 1 \boldsymbol{k}$-meshes for self-consistent electronic structure calculations of graphene adsorbed on h-BN thin films. To calculate the hybrid structure composed of graphene and h-BN, we assumed a commensurability condition between their lateral lattice parameter of $2.46 \AA$, which corresponds to the experimental value of graphene, because the electronic states of graphene are responsible for carrier doping. All atoms were fully optimized until the remaining force acting on each atom was less than $0.005 \mathrm{Ry} / \AA$ under the fixed lateral lattice constant.

In this work, we considered graphene FETs with h-BN thin films as dielectric layers used as the gate electrode (Fig. 1). During the calculations under a finite gate voltage, we assumed that the atomic structure of graphene and h-BN were fixed at the optimized structure obtained under zero electric field. Electrons and holes were injected by a planar gate electrode situated above the h-BN thin film with a vacuum spacing of $4.0 \AA$, mimicking a single-gate graphene FET. The electrode was simulated using an ESM with infinite relative permittivity. 


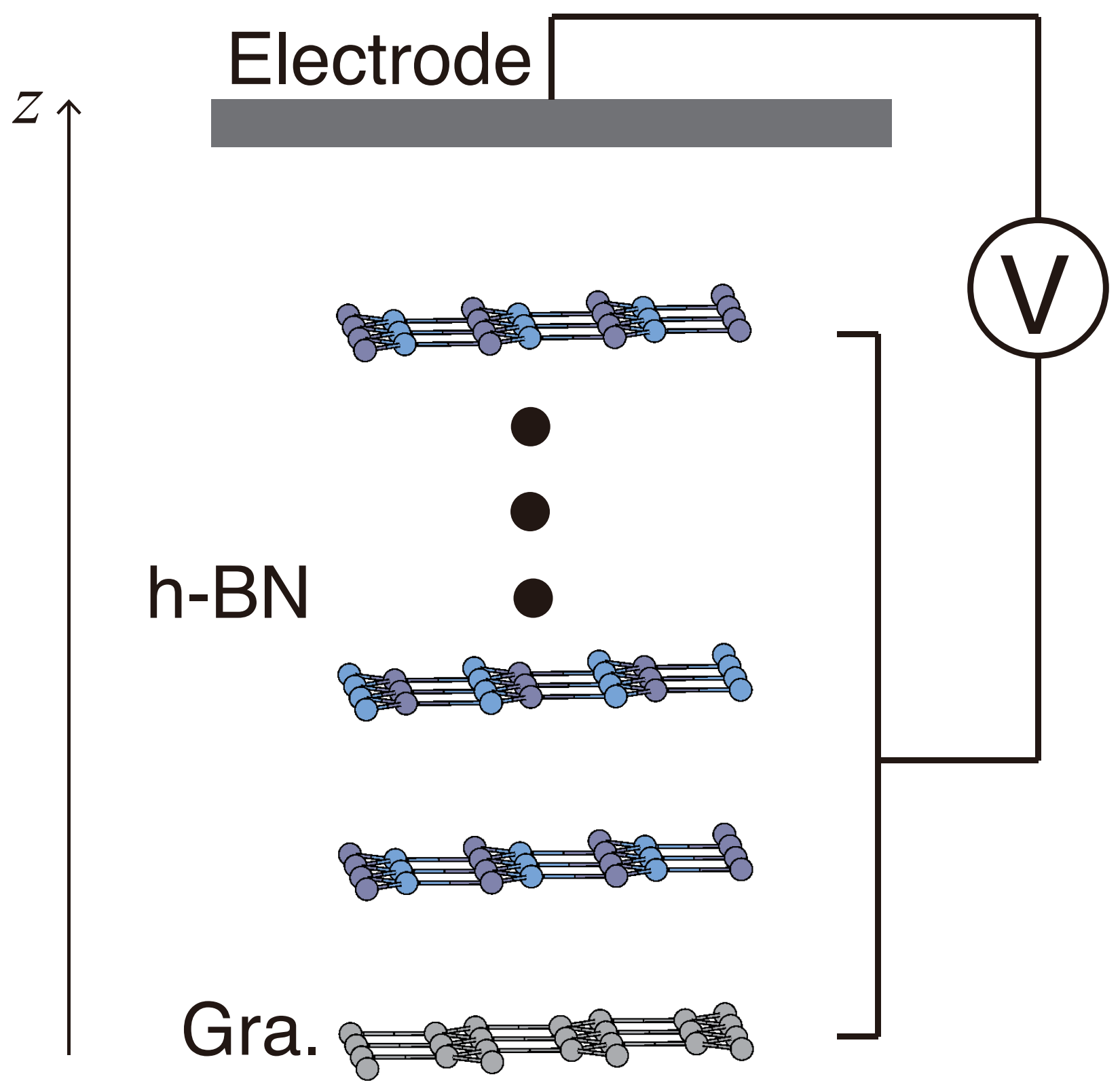

FIG. 1. Structural model of a graphene FET with an h-BN thin film. Gray, blue, and cyan circles denote $\mathrm{C}, \mathrm{N}$, and $\mathrm{B}$ atoms, respectively. The gray rectangle indicates the counter electrode with infinite relative dielectric constant simulated by the effective screening medium.

Figure 2 shows the carrier distribution in graphene FETs with monolayer h-BN dielectrics as a function of the $z$ axis, corresponding to the normal direction to the layers, under gate voltages of 1 and $4 \mathrm{~V}$, which correspond to carrier densities of $1.9 \times 10^{13} \mathrm{e}(\mathrm{h})$ and $7.6 \times 10^{13} \mathrm{e}(\mathrm{h}) / \mathrm{cm}^{2}$, respectively. Under low electron and hole concentrations, distributions of 
(a)

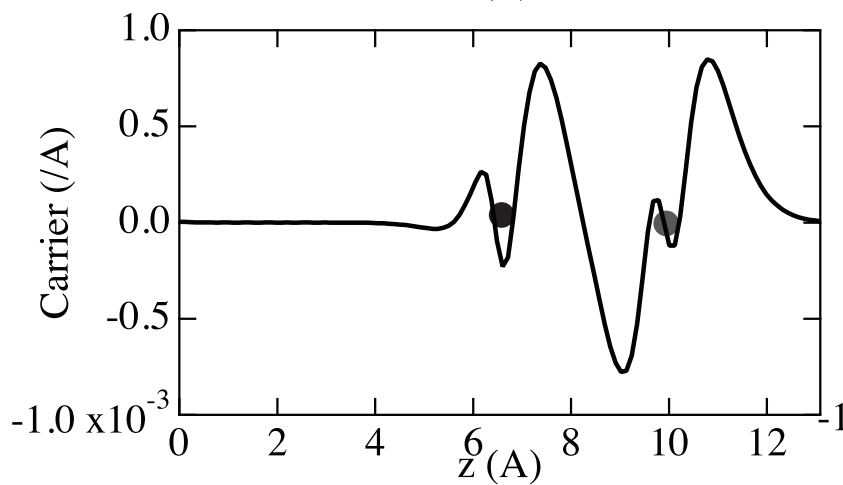

(c)

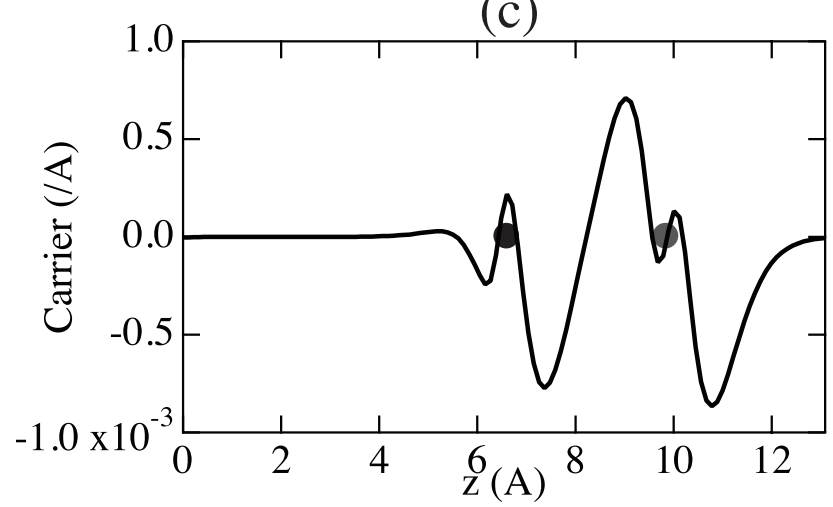

(b)

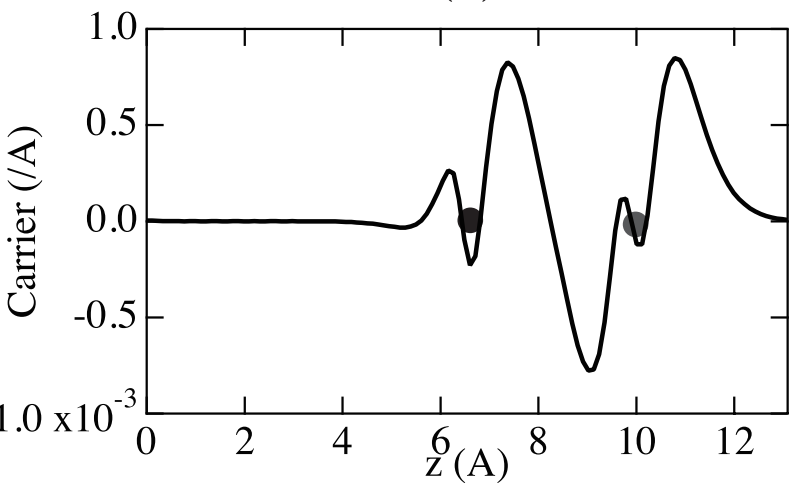

(d)

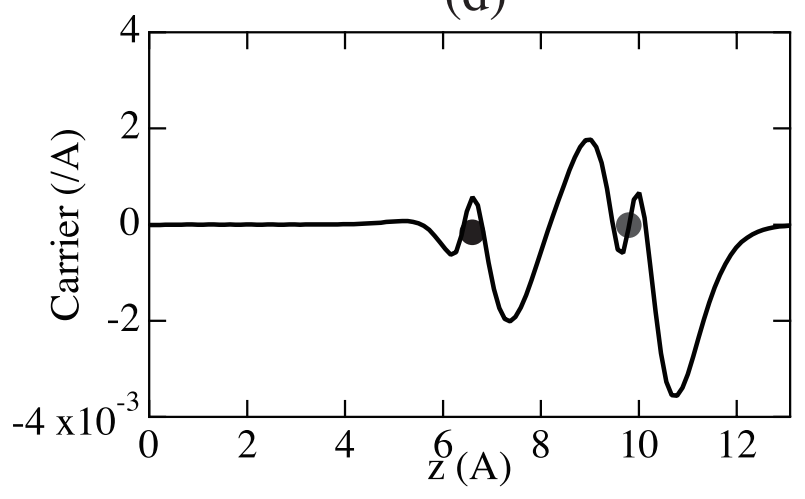

FIG. 2. Charge density across the layers of graphene FETs with monolayer h-BN under gate voltages of (a) 1, (b) 4, (c) -1, and (d) $-4 \mathrm{~V}$ corresponding to doping concentrations of $1.9 \times 10^{13} \mathrm{e}$, $7.6 \times 10^{13} \mathrm{e}, 1.9 \times 10^{13} \mathrm{~h}$, and $7.6 \times 10^{13} \mathrm{~h} / \mathrm{cm}^{2}$, respectively. Filled circles indicate the atomic positions of graphene and h-BN. The counter electrode is located on the right-hand side of each figure.

accumulated carriers exhibited symmetric behavior in terms of the carrier species. Counter carriers on the electrode caused polarization across the h-BN layer and carrier accumulation in the graphene layer. Electron density increased and decreased on the electrode-side and graphene-side surfaces of h-BN, respectively. Electron density increased on both sides of graphene under electron doping. A similar carrier distribution was also observed in the case of hole doping. Integrating the carrier density along the $z$ axis, approximately $70 \%$ and $65 \%$ of carriers were accommodated in the graphene layer under electron and hole doping, respectively. In addition, asymmetric polarization across the h-BN layer caused carriers to accumulate in the h-BN layer. 


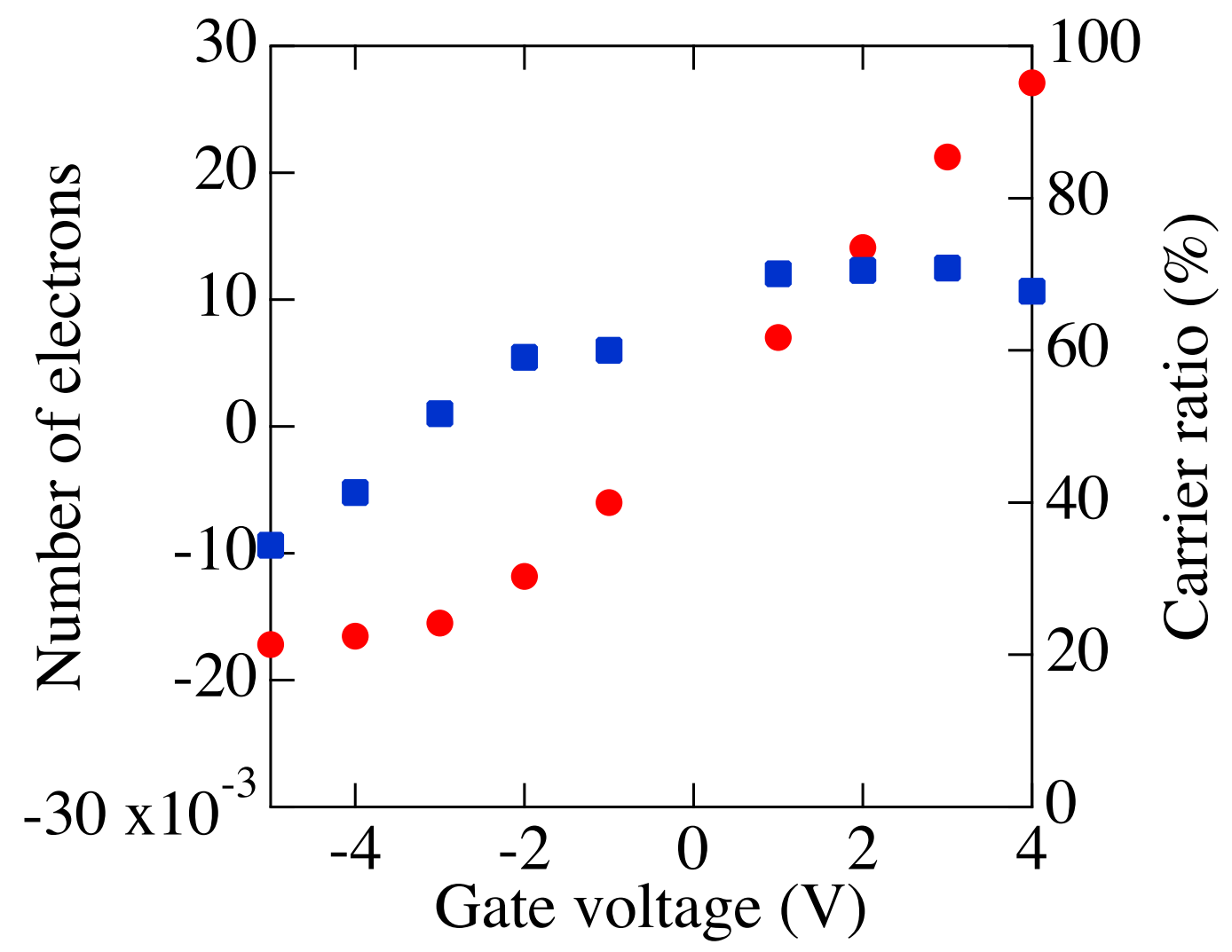

FIG. 3. Number of carriers accommodated in the graphene layer (circles) and its ratio with the number of carriers injected by the gate voltage (squares) as a function of the gate voltage.

At high carrier concentration, the carrier distribution across the graphene and h-BN layers was asymmetric in terms of the carrier species. In the case of electron doping, approximately $70 \%$ of the electrons were accommodated in the graphene layer, while the rest were accommodated in h-BN because of the asymmetric polarization. Under hole doping, injected holes were mainly accommodated in h-BN. Integrating the carrier density along the $z$ axis, $60 \%$ of holes were injected into the h-BN layer, indicating carrier penetration into h-BN at that doping level.

Figure 3 shows the number of carriers and its ratio with the number of doped carriers in graphene as a function of the gate voltage. The carrier density in graphene was proportional to the positive gate voltage, leading to a constant carrier ratio of approximately $70 \%$ in graphene. In contrast, hole density was saturated at a density of $3 \times 10^{13} \mathrm{e} / \mathrm{cm}^{2}$ at a gate voltage of $-4 \mathrm{~V}$, which prevented further hole injection into graphene. Therefore, h-BN 
(a)

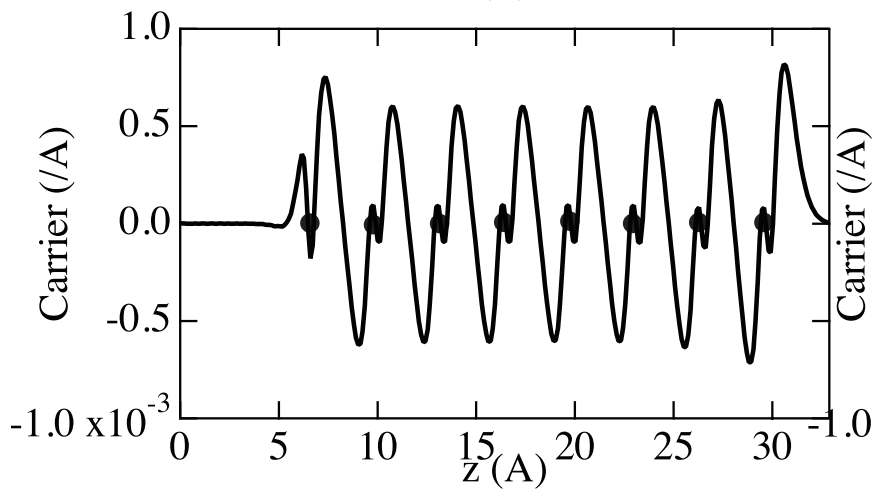

(b)

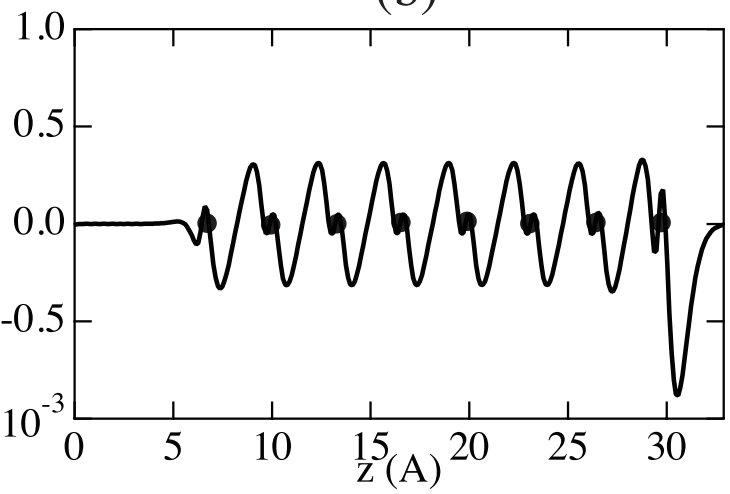

FIG. 4. Charge density across the layers of a graphene FET with an h-BN gate dielectric composed of seven layers under gate voltages of (a) 1 and (b) $-1 \mathrm{~V}$ corresponding to doping concentrations of $1.9 \times 10^{13} \mathrm{e}$ and $1.9 \times 10^{13} \mathrm{~h} / \mathrm{cm}^{2}$, respectively. Filled circles indicate the atomic positions of graphene and h-BN. The counter electrode is located on the right-hand side of each figure.

exhibited asymmetric field screening ability in terms of the gate voltage.

Next, we investigated the influence of h-BN thickness on the carrier accumulation in graphene. Figure 4 shows the distribution of accumulated carriers across graphene and h-BN layers when the dielectric consisted of seven layers. Under a positive gate voltage corresponding to a carrier concentration of $1.9 \times 10^{13} \mathrm{e} / \mathrm{cm}^{2}$, graphene accommodated $70 \%$ of electrons injected by the field. The rest of the carriers were distributed on the topmost layer of the h-BN thin film because of the asymmetric polarization induced by the counter carrier on the electrode. In contrast, no carriers were observed in the second to the sixth sublayers of h-BN. Symmetric polarization was observed in each of these layers. Under hole doping with a carrier concentration of $1.9 \times 10^{13} \mathrm{~h} / \mathrm{cm}^{2}$, the carrier distribution in the graphene FET with an h-BN thin film exhibited different behavior to that determined under electron doping. Even at low hole concentration, $70 \%$ of the holes injected by the field were accommodated in the topmost layer of h-BN. Only $30 \%$ of holes were accommodated in the graphene layer.

The dependence of the carrier density in graphene and the topmost layer of h-BN on the hBN thickness were investigated (Fig. 5). Under an excess electron density of $1.9 \times 10^{13} \mathrm{e} / \mathrm{cm}^{2}$, approximately $70 \%$ of electrons were distributed in graphene while the remaining $30 \%$ were distributed in the topmost layer of h-BN irrespective of the h-BN thickness. In contrast, 
(a)

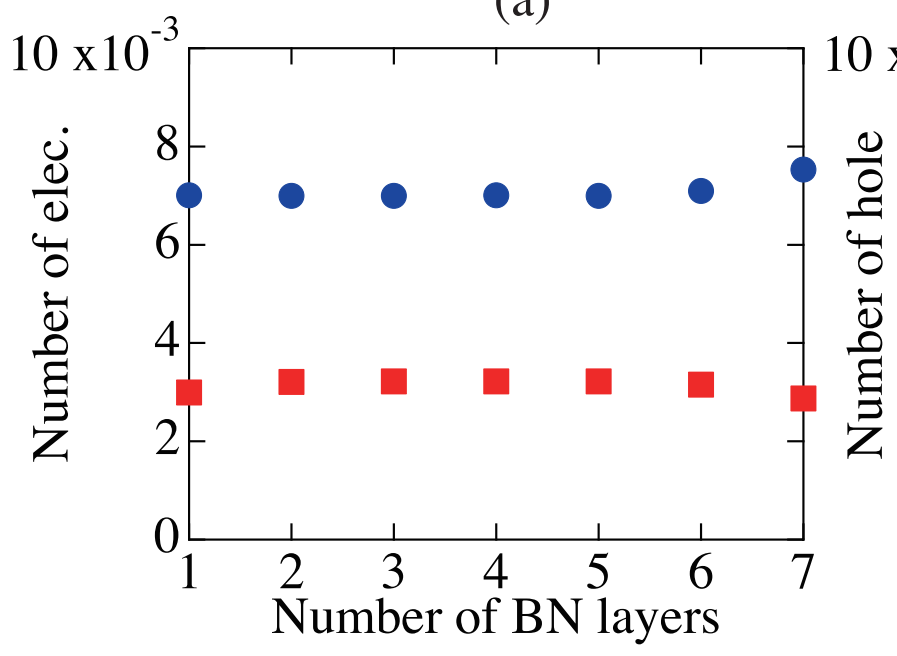

(b)

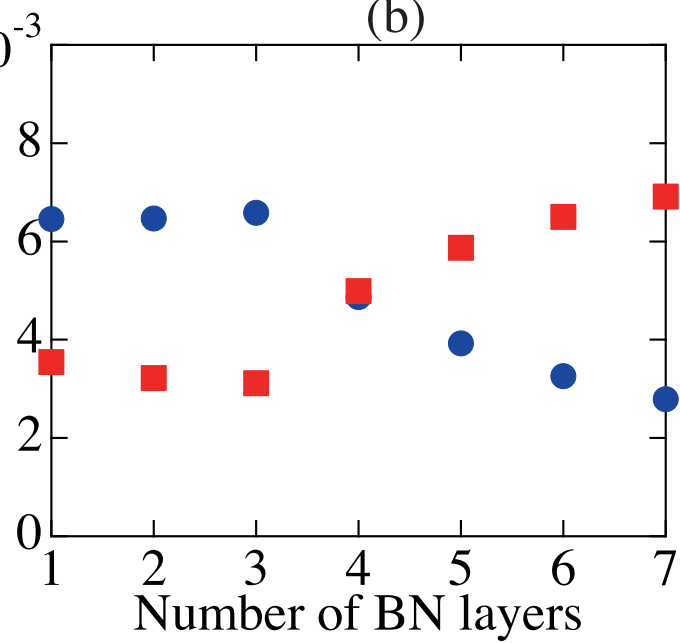

FIG. 5. Number of carriers in graphene (circles) and the topmost layer of h-BN thin films (squares) as a function of h-BN film thickness under gate voltages of (a) 1 and (b) $-1 \mathrm{~V}$, corresponding to $1.9 \times 10^{13} \mathrm{e}$ and $1.9 \times 10^{13} \mathrm{~h} / \mathrm{cm}^{2}$ doping, respectively.

the hole distribution strongly depended on h-BN thickness under the doping concentration of $1.9 \times 10^{13} \mathrm{~h} / \mathrm{cm}^{2}$, owing to the potential difference among the h-BN layers arising from the perpendicular electric field. The injected holes were primarily accommodated in graphene up to trilayer h-BN, where $70 \%$ and $30 \%$ of holes were distributed in graphene and the topmost layer of h-BN, respectively. For four-layer h-BN, doped holes were equally distributed in graphene and the topmost layer of h-BN. For the thicker h-BN films (five or more layers), the hole density in the topmost h-BN layer was higher than that in graphene, and monotonically increased with h-BN thickness up to the seven layers studied here. Therefore, h-BN dielectric layers are less stable against the hole doping than the electron doping in terms of carrier concentration and film thickness.

The asymmetric carrier penetration into h-BN thin films in terms of carrier species was ascribed to the band edge alignment of graphene and h-BN. The eigenvalue of the valence band top of graphene and $n$-layer h-BN, and that of the conduction band bottom of graphene and $n$-layer h-BN are summarized in Table I. The valence band top of monolayer h-BN is located $1.6 \mathrm{eV}$ below the Dirac point, whereas the conduction band bottom is located 3 $\mathrm{eV}$ above the Dirac point. This indicates that the Fermi level is closer to the valance band edge than to the conduction band edge of h-BN. The eigenvalue of the valence and 
TABLE I. The eigenvalue of the valence band top of graphene $\left(\mathrm{VT}_{\mathrm{G}}\right)$ and of $n$-layer h-BN $\left(\mathrm{VT}_{\mathrm{BN}}\right)$, and that of the conduction band bottom of graphene $\left(\mathrm{CB}_{\mathrm{G}}\right)$ and of $n$-layer h-BN $\left(\mathrm{CB}_{\mathrm{BN}}\right)$. Energies are measured from the valence band top $\left(\mathrm{VT}_{\mathrm{G}}\right)$. Note that the $\mathrm{VT}_{\mathrm{G}}$ and $\mathrm{CB}_{\mathrm{G}}$ do not degenerate each other because of the interaction between graphene and underneath h-BN thin films ${ }^{41}$.

\begin{tabular}{lcccc}
\hline \hline & $\mathrm{VT}_{\mathrm{BN}}(\mathrm{eV})$ & $\mathrm{VT}_{\mathrm{G}}(\mathrm{eV})$ & $\mathrm{CB}_{\mathrm{G}}(\mathrm{eV})$ & $\mathrm{CB}_{\mathrm{BN}}(\mathrm{eV})$ \\
\hline Gra/1L-h-BN & -1.607 & 0.000 & 0.077 & 3.110 \\
Gra/2L-h-BN & -1.566 & 0.000 & 0.073 & 3.116 \\
Gra/3L-h-BN & -1.561 & 0.000 & 0.076 & 3.089 \\
Gra/4L-h-BN & -1.566 & 0.000 & 0.073 & 3.085 \\
Gra/5L-h-BN & -1.565 & 0.000 & 0.073 & 3.079 \\
Gra/6L-h-BN & -1.563 & 0.000 & 0.075 & 3.082 \\
Gra/7L-h-BN & -1.573 & 0.000 & 0.068 & 3.073 \\
\hline \hline
\end{tabular}

conduction band edges of h-BN is insensitive to the number of layers up to the seven layers studied here. Note that the conduction band edge of h-BN shown here is expected to be deeper than that obtained by the state-of-the-art procedures, such as GW approximation and hybrid exchange-correlation functional, because of the choice of the LDA exchangecorrelation functional. Accordingly, the critical voltage for hole penetration is lower than that for electron penetration. Furthermore, the relative dielectric constant of h-BN ranged from 2.2 to 2.6, slightly increasing with film thickness from monolayer to seven layers. This trend indicates that $\mathrm{h}-\mathrm{BN}$ is unlikely to be suitable for practical use as a dielectric layer in graphene FETs, even though it is a good supporting layer for measuring the transport properties of graphene.

In conclusion, the electronic structure of graphene FETs with h-BN thin films under external electric fields was investigated in terms of gate electric field and h-BN film thickness using DFT combined with the ESM method. Asymmetric carrier penetration into the topmost h-BN layer with respect to the carrier species occurred because of the band edge alignment of h-BN thin films to the Dirac point of graphene. For monolayer h-BN under a weak gate voltage, approximately $70 \%$ of carriers were accommodated in the graphene layer and $30 \%$ of carriers were distributed in h-BN because of the asymmetric polarization induced by the counter charge on the electrode. In contrast, under a strong gate voltage, 
$70 \%$ of electrons were accommodated in graphene, whereas most holes spilled over to the topmost h-BN layer, indicating that injected carrier is spilled over h-BN layer at high hole concentration. For the case of graphene with h-BN films with different thickness under a hole concentration of $1.9 \times 10^{13} \mathrm{~h} / \mathrm{cm}^{2}$, the carrier spill started at four-layer h-BN and the concentration of spilled holes rose monotonically with the number of h-BN layers. The thin films of all thicknesses studied retained their dielectric properties under an electron concentration of $1.9 \times 10^{13} \mathrm{e} / \mathrm{cm}^{2}$.

\section{ACKNOWLEDGMENTS}

The author thanks JST-CREST Grant Numbers JPMJCR1532 and JPMJCR1715 from the Japan Science and Technology Agency, JSPS KAKENHI Grant Numbers JP20K05253, JP20H02080, JP20H00316, JP17H01069, and JP16H06331 from the Japan Society for the Promotion of Science, the Joint Research Program on Zero-Emission Energy Research, Institute of Advanced Energy, Kyoto University, and University of Tsukuba Basic Reseach Support Program (S). Part of the calculations was performed on an NEC SX-Ace at the Cybermedia Center at Osaka University.

\section{REFERENCES}

${ }^{1}$ M. S. Dresselhaus and G. Dresselhaus, Adv. Phys. 30, 139 (1981).

${ }^{2}$ A. H. Castro Neto, F. Guinea, N. M. R. Peres, K. S. Novoselov, and A. K. Geim, Rev. Mod. Phys. 81, 109 (2009).

${ }^{3}$ G. S. Painter and D. E. Ellis, Phys. Rev. 1, 4747 (1970).

${ }^{4}$ F. Bassani and G. P. Parravicini, Nuovo Cimento B 50, 95 (1967).

${ }^{5}$ M. Posternak, A. Baldereschi, A. J. Freeman, E. Wimmer and M. Weinert, Phys. Rev. Lett. 50, 761 (1983).

${ }^{6}$ K. S. Novoselov, A. K. Geim, S. V. Morozov, D. Jiang, M. I. Katsnelson, I. V. Grigorieva, S. V. Dubonos, and A. A. Firsov, Nature 438, 197 (2005).

${ }^{7}$ Y. Zhang, Y.-W. Tan, H. L. Stormer, and P. Kim, Nature 438, 201 (2005).

${ }^{8}$ K. I. Bolotin, K. J. Sikes, Z. Jiang, M. Klima, G. Fudenberg, J. Hone, P. Kim, and H. L. Stormer, Solid State Commun. 146, 351 (2008). 
${ }^{9}$ Y. Ma, P. O. Lehtinen, A. S. Foster, and R. M. Nieminen, New J. Phys. 6, 68 (2004).

${ }^{10}$ H. Amara, S. Latil, V. Meunier, Ph. Lambin, and J.-C. Charlier, Phys. Rev. B 76, 115423 (2007).

${ }^{11}$ M. M. Ugeda, I. Brihuega, F. Hiebel, P, Mallent, J.-Y. Veuillen, J. M. G.-Rodriguez, and F. Yndurain, Phys. Rev. B 85, 121402(R) (2012).

${ }^{12}$ M. Maruyama, N. T. Cuong, and S. Okada, Carbon 109, 755 (2016).

${ }^{13}$ J. Lahiri, Y. Lin, P. Bozkurt, I.I. Oleynik, and M. Batzill, Nat. Nanotechnol. 5, 326 (2010).

${ }^{14}$ S. Okada, T. Kawai, and K. Nakada, J. Phys. Soc. Jpn. 80, 013709 (2011).

${ }^{15}$ M. Maruyama and S. Okada, Appl. Phys. Express 6, 095101 (2013).

${ }^{16}$ M. Fujita, K. Wakabayashi, K. Nakada, and K. Kusakabe, J. Phys. Soc. Jpn. 65, 1920 (1996).

${ }^{17}$ K. Nakada, M. Fujita, G. Dresselhaus, and M. S. Dresselhaus, Phys. Rev. B 54, 17954 (1996).

${ }^{18}$ P. Koskinen, S. Malola, and H. Hakkinen, Phys. Rev. Lett. 101, 115502 (2008).

${ }^{19}$ N. Igami, K. Nakada, and S. Okada, Synth. Met. 121, 1233(2001).

${ }^{20}$ R. Balog, B. Jorgensen, L. Nilsson, M. Andersen, E. Rienks, M. Bianchi, M. Fanetti, E. Laegsgaard, A. Baraldi, S. Lizzit, Z. Sljivancanin, F. Besenbacher, B. Hammer, T. G. Pedersen, P. Hofmann, and L. Hornekaer, Nat. Mater. 9, 315 (2010).

${ }^{21}$ M. Koshino and T. Ando, Phys. Rev. B 76, 085425 (2007).

${ }^{22}$ E. Suárez Morell, J. D. Correa, P. Vargas, M. Pacheco, and Z. Barticevic, Phys. Rev. B 82, 121407(R) (2010).

${ }^{23}$ R. Bistritzer and A. H. MacDonald, Proc. Natl. Acad. Sci. 108, 12233 (2011).

${ }^{24}$ Y. Cao, V. Fatemi, S. Fang, K. Watanabe, T. Taniguchi, E. Kaxiras, and P. Jarillo-Herrero, Nature 556, 43 (2018).

${ }^{25}$ S. Adam, E.H. Hwang, V.M. Galitski, and S. Das Sarma, Proc. Natl. Am. Soc. 104, 18392 (2007).

${ }^{26}$ J.-H. Chen, C. Jang, M. S. Fuhrer E. D. Williams, and M. Ishigami: Nat. Phys. 4, 377 (2008).

${ }^{27}$ J.-H. Chen, C. Jang, S. Xiao, M. Ishigami, and M.S. Fuhrer : Nat. Nanotechnol. 3, 206 (2008).

${ }^{28}$ X. Hong, K. Zou, and J. Zhu : Phys. Rev. B 80, 241415(R) (2009). 
${ }^{29}$ K. Nagashio, T. Yamashita, T. Nishimura, K. Kita, and A. Toriumi, J. Appl. Phys. 110, 024513 (2011).

${ }^{30}$ N. T. Cuong, M. Otani, and S. Okada, Phys. Rev. 106, 106801 (2011).

${ }^{31}$ K. Kamiya, N. Umezawa, and S. Okada, Phys. Rev. B 83, 153413 (2011).

${ }^{32}$ C. R. Dean, A. F. Young, I. Meric, C. Lee, L. Wang, S. Sorgenfrei, K. Watanabe, T. Taniguchi, P. Kim, K. L. Shepard, and J. Hone, Nat. Nanotechnol. 5, 722 (2010).

${ }^{33}$ A. Catellani, M. Posternak, A. Baldereschi, and A. J. Freeman, Phys. Rev. B 36, 6105 (1987).

${ }^{34}$ X. Blase, A. Rubio, S. G. Louie, and M. L. Cohen, Phys. Rev. B .51, 6868 (1995).

${ }^{35}$ P. Hohenberg and W. Kohn, Phys. Rev. 136, B864 (1964).

${ }^{36}$ W. Kohn and L. J. Sham, Phys. Rev. 140, A1144 (1965).

${ }^{37}$ Y. Morikawa, K. Iwata, and K. Terakura, Appl. Surf. Sci. 169-170, 11 (2001).

${ }^{38}$ J. P. Perdew and A. Zunger, Phys. Rev. B 23, 5048 (1981).

${ }^{39}$ D. M. Ceperley and B. J. Alder, Phys. Rev. Lett. 45, 566 (1980).

${ }^{40}$ S. Okada, S. Saito, and A. Oshiyama, Phys. Rev. Lett. 86 , 3835 (2001).

${ }^{41}$ Y. Sakai, T. Koretsune, and S. Saito, Phys. Rev. B 83, 205434 (2011).

${ }^{42}$ D. Vanderbilt, Phys. Rev. B 41, 7892 (1990).

${ }^{43}$ M. Otani and O. Sugino, Phys. Rev. B 73, 115407 (2006).

${ }^{44}$ M. Maruyama and S. Okada, Appl. Phys. Express 12, 075008 (2019). 